\begin{tabular}{|c|l|}
\hline Title & $\begin{array}{l}\text { Nitric Oxide Produced During I schemia Is Toxic but Crucial to Preconditioning-Induced Ischemic Tolerance of } \\
\text { Neurons in Culture }\end{array}$ \\
\hline Author(s) & Kawahara, Koichi; Y anoma, Junji; Tanaka, Motoki; Nakajima, Takay uki; Kosugi, Tatsuro \\
\hline Citation & $\begin{array}{l}\text { Neurochemical Research, 29(4), 797-804 } \\
\text { https://doi.org/10.1023/B:NERE.0000018853.30131.4d }\end{array}$ \\
\hline Issue Date & 2004-04 \\
\hline Doc URL & http://hdl.handle.net/2115/5933 \\
\hline Rights & $\begin{array}{l}\text { The original publication is avail lable at www.springerlink.com. } \\
\text { Former publisher: Kluwer A cademic Publishers. }\end{array}$ \\
\hline Type & article (author version) \\
\hline File Information & NR29-4.pdf \\
\hline
\end{tabular}

Instructions for use 


\section{Nitric oxide produced during ischemia is toxic but crucial to preconditioning-induced ischemic tolerance of neurons in culture.}

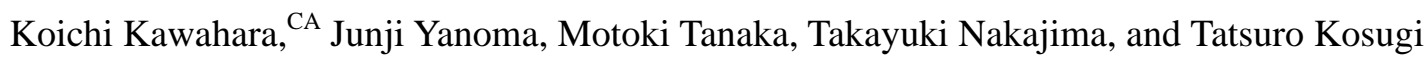

Laboratory of Biomedical Control, Research Institute for Electronic Science, Hokkaido University, Sapporo 060-0812, Japan

Running title: ischemic preconditioning and nitric oxide

Address correspondence to:

Koichi Kawahara, PhD.

Professor of the Laboratory of Biomedical Control

Research Institute for Electronic Science

Hokkaido University

Sapporo 060-0812

Japan

TEL \& FAX: +81-11-706-2893

E-mail: kawahara@bmc.es.hokudai.ac.jp 


\section{Abstract}

The present study investigated the roles of nitric oxide (NO) in preconditioning

(PC)-induced neuronal ischemic tolerance in cortical cultures. Ischemia in vitro was simulated by subjecting cultures to both oxygen and glucose deprivation (OGD). A sublethal OGD (PC) significantly increased the survival rate of neurons when cultures were exposed to a lethal OGD 24 hr later. Both the inhibition of nitric oxide synthase (NOS) and scavenging of NO during PC significantly attenuated the PC-induced neuronal tolerance. In addition, exposure to an NO donor emulated the PC. In contrast, the inhibition of NOS and the scavenging of NO during lethal OGD tended to increase the survival rate of neurons. This study suggested that NO produced during ischemia was fundamentally toxic, but critical to the development of PC-induced neuronal tolerance.

Key words: ischemia, preconditioning, nitric oxide, neurotoxicity, cortical culture 


\section{Introduction}

Preconditioning (PC) to ischemic tolerance is a phenomenon in which a brief subtoxic

insult induces robust protection against the deleterious effects of a subsequent, prolonged, lethal

ischemia (1). In the brain, Kitagawa et al. (2) first reported that gerbils subjected to a sublethal transient global ischemia exhibited reduced hippocampal neuronal death after a more severe ischemic insult 24 28 hr later. Since then, numerous studies have been performed on this phenomenon, but the mechanistic basis of PC-induced ischemic tolerance has not been fully delineated. For the induction of ischemic tolerance, a triggering role of neuronal NMDA receptor activation has been suggested $(3,4)$. We have recently found that the PC-induced immediate enhancement in the phosphorylation of CREB in the penumbra region during subsequent lethal ischemia is crucially involved (5), although the triggering mechanisms for the enhancement remain unknown. Cinani et al. (6) recently demonstrated that nitric oxide (NO) signaling is functionally coupled to the phosphorylation of CREB, and is involved in the survival of neurons.

NO is generated in various mammalian tissues, and acts as an intercellular messenger associated with various physiological and pathological events (7). Recently, we have 
demonstrated that the activity of nitric oxide synthase (NOS) varies associated with the differentiation of a neuronal cell line (NG108-15 cells), suggesting that NO functions as an important signaling molecule in differentiated NG108-15 cells (8). We have also found that NO modulates the propagation of astrocytic $\mathrm{Ca}^{2+}$ waves induced by the local photolysis of caged $\mathrm{Ca}^{2+}$ ionophore $(9,10)$. In addition, Yun et al. (11) have recently demonstrated that the activation of $\mathrm{p} 21^{\mathrm{RAS}}$ induced by NO derived from nNOS is responsible for the PC-induced tolerance of neurons, suggesting that $\mathrm{NO}$ is a key mediator in the process leading to tolerance against lethal ischemia.

Here we provide experimental evidence suggesting that NO produced during ischemia was fundamentally toxic to neurons, but critical to the development of PC-induced ischemic tolerance of neurons in culture. 


\section{Experimental Procedure}

The animal experiments conformed to the "Principles of laboratory animal care" (NIH publication No. 85-23, revised 1996), as well as the "guide for the care and use of laboratory animals”, Hokkaido University School of Medicine (Hokkaido, Japan).

\section{Cell culture.}

Culture methods were described elsewhere in detail (12). In brief, neurons were prepared

from 16 to 18 day old embryonic rat cortices and grown in Dulbecco's modified Eagle’s medium (DMEM, Gibco, Grand Island, NY) which was supplemented with $10 \%$ heat-inactivated fetal bovine serum (FBS), 10 \% Ham’s F12, and $0.24 \%$ penicillin/streptomycin (culture medium). Cells were plated at a uniform density of $3.0 \times 10^{5}$ cells $/ \mathrm{cm}^{2}$ onto poly-L-lysine (100 $\mu \mathrm{g} / \mathrm{ml})$-coated plastic dishes and maintained in a $5 \% \mathrm{CO}_{2}$ incubator at $37{ }^{\circ} \mathrm{C}$. The cultures were fed a filtered $(0.22 \mu \mathrm{m}$; Millipore, Bedford, TX) conditioned medium (CM) twice a week. To obtain the CM, cells from the 16-18 day old embryonic rat cortices were plated onto poly-L-lysine-coated 6-well dishes and cultured for more than 2 weeks.

The cultures were then fed a cooled culture medium and incubated for an additional day. The 
culture medium was then filtered and used as a CM. The experiments described here were performed on neurons maintained for 13-15 days in culture.

\section{Immunocytochemistry.}

Neurons were identified by immunostaining with an antibody against microtubule-associated protein 2 (MAP-2; Sigma, St Louis, MO). The nitric oxide synthases (NOS) in cultured cells were detected by immunostaining with anti-neuronal NOS (nNOS) (Euro-Diagnostica, Sweden), anti-endothelial NOS (eNOS) (Transduction Lab., Lexington KY), or anti-inducible NOS (iNOS) (Transduction Lab.). For the labeling of MAP-2, nNOS, eNOS, and iNOS, the cortical cells were fixed with $4 \%$ paraformaldehyde for 5 minutes at $4{ }^{\circ} \mathrm{C}$, followed by $95 \%$ methanol in PBS for 10 minutes at $-20{ }^{\circ} \mathrm{C}$. The cells were then incubated with a primary antibody over a 24 hour period using a dilution of 1:1,000 for MAP-2, 1:1,000 for nNOS, 1:1,000 for eNOS, and 1:5,000 for iNOS. After being washed with phosphate-buffered saline (PBS), the cells were incubated with a secondary antibody containing $1.0 \%$ goat serum for 30 minutes. For labeling, a 1:500 dilution of biotinylated goat antibody against mice IgG (Vector Laboratories, Burlingame, CA) was used. Bound antibodies were 
detected by the avidin-biotin-peroxidase complex (ABC) method, using a commercial ABC kit

(Vector Laboratories). Observation of peroxidase activity was made possible by incubation

with $0.1 \%$ 3,3'-diaminobenzidine tetrahydrochloride (DAB) in a $50 \mathrm{mM}$ Tris- $\mathrm{HCl}$ buffer (pH

7.4) supplemented with $0.02 \% \mathrm{H}_{2} \mathrm{O}_{2}$. The cells were dehydrated in $70-100 \%$ ethanol, cleared in xylene, and mounted on glass coverslips in Permount (Fisher Scientific, Fair Lawn, NJ) for light microscopic observation.

\section{Oxygen-Glucose deprivation.}

Cortical cultures were subjected to oxygen-glucose deprivation (OGD) injury using a protocol described previously (13). In brief, cultures were placed in an anaerobic chamber and washed two times with balanced salt solution (BSS: $116 \mathrm{mM} \mathrm{NaCl}, 0.8 \mathrm{mM} \mathrm{MgSO}$, $5.4 \mathrm{mM}$ $\mathrm{KCl}, 1.0 \mathrm{mM} \mathrm{NaH}{ }_{2} \mathrm{PO}_{4}, 26.2 \mathrm{mM} \mathrm{NaHCO} 3,1.8 \mathrm{mM} \mathrm{CaCl}_{2}, 0.01 \mathrm{mM}$ glycine, and $10 \mathrm{mg} / \mathrm{l}$ phenol red) lacking glucose. Near anoxic conditions were achieved using an Anaero-Pack System (Mitsubishi Gas Chemical, Tokyo, Japan). After pre-gassing with $95 \% \mathrm{~N}_{2}-5 \% \mathrm{CO}_{2}$ for at least 5 min to remove residual oxygen, glucose-free BSS was added to the cells, which were then placed in a purpose-built sealed chamber containing the deoxygenation reagent 
(Kenki for Cells, Mitsubishi Gas Chemical). The catalytic reaction of the reagent resulted in the consumption of $\mathrm{O}_{2}$ and production of $\mathrm{CO}_{2}$. This Anaero-Pack System provided near anaerobic conditions with an $\mathrm{O}_{2}$ concentration of $<1 \%$ and a $\mathrm{CO}_{2}$ concentration of about $5 \%$ within $1 \mathrm{~h}$ of incubation at $37{ }^{\circ} \mathrm{C}$. Cells were exposed to these conditions for a designated period to produce either mild (sublethal) or lethal OGD. To terminate OGD, cultures were carefully washed with glucose $(20 \mathrm{mM})$ containing DMEM, and then incubated again at $37{ }^{\circ} \mathrm{C}$ in $95 \%$ air-5 \% $\mathrm{CO}_{2}$ (reperfusion). Cultures with sham treatment not deprived of oxygen and glucose were placed in BSS containing $20 \mathrm{mM}$ glucose.

\section{Survival rate of neurons.}

Neuronal death was analyzed following observation of the nuclear morphology using the fluorescent DNA-binding dyes, Hoechst 33342 (H33342) and propidium iodide (PI). Cells were incubated with these dyes for 15 minutes at $37^{\circ} \mathrm{C}$. Individual nuclei were observed using fluorescent microscopy (Olympus, IX70, Tokyo, Japan) and subsequently analyzed. PI was used to identify nonviable cells. More specifically, an average of 450-500 neurons from random fields were analyzed in each experiment. The survival rate of neurons -- meaning the 
percentage of viable neurons remaining -- was determined by placing images of nuclear staining on phase-contrast images, and calculating (viable neurons/total neurons before drug treatment) $\times 100$, since some neurons came off the dishes at the time of inspection. At least 4 independent experiments ( $\mathrm{n} \geqq 4$ ) were conducted and analyzed.

\section{Chemicals.}

$\mathrm{N}^{\mathrm{G}}$-monomethyl-L-arginine (L-NMMA), Bisbenzimide (Hoechst 33342), and propidium iodide were

obtained

from

Sigma.

2-(4-Carboxyphenyl)-4,4,5,5-tetramethylimidazoline-1-oxyl3-oxide (Carboxy-PTIO), and S-nitroso-N-acetyl-DL-penicillamine (SNAP) were obtained from Dojindo Lab., Inc. (Kumamoto, Japan). The other chemicals were from Wako Chem. (Tokyo, Japan).

\section{Statistics.}

Data are represented as the mean \pm S.D. Inter-group comparisons were made using the one-way analysis of variance (ANOVA) followed by a paired t-Test. Differences with a value of $\mathrm{P}<0.05$ were considered significant. 


\section{Results}

We first investigated whether sublethal oxygen-glucose deprivation (OGD) induced

neuronal ischemic tolerance in murine cortical cultures containing both neurons and glia (days

in vitro 13-15). To do this, we have tried to determine both the sublethal and lethal exposure

periods of OGD for cultured neurons (Fig. 1). Exposure to OGD for $1 \mathrm{hr}$ did not result in

significant neuronal death when examined $24 \mathrm{hr}$ later (Fig. 1C and E). However, exposure for

$2 \mathrm{hr}$ produced massive neuronal death without glial degeneration $24 \mathrm{hr}$ later (Fig. 1D and E).

Thus, we adopted the $1 \mathrm{hr}$ OGD as the preconditioning (PC), and 2hr OGD as the lethal insult.

We then tried to determine the time interval between the PC and the lethal OGD for the effective

development of ischemic tolerance in neurons. Cultures were preconditioned with $1 \mathrm{hr}$ OGD

and then exposed to lethal (2 hr) OGD 6, 24, and $48 \mathrm{hr}$ later (Fig. 1F). Exposure of

preconditioned cultures to lethal OGD $24 \mathrm{hr}$ later resulted in a significant increase in the

survival rate of neurons inspected $24 \mathrm{hr}$ after the end of the lethal insult. However, no

significant protective effect on neuronal death was observed in the preconditioned cultures

exposed to the lethal insult 6 and 48 hr later. Thus, the time interval between the PC and the

lethal OGD was set at $24 \mathrm{hr}$ (reperfusion period). 
We then investigated whether nitric oxide (NO) is involved in the development of PC-induced ischemic tolerance in neurons. We first performed an immunohistochemical analysis to delineate the expression patterns of nitric oxide synthase (NOS) isoforms (Fig. 2A). nNOS proteins were detected in the cultured neurons (A1), but neither iNOS (A2) nor eNOS (A3) proteins were clearly identified. We next analyzed whether the PC-induced ischemic tolerance of neurons was attenuated when the activity of NOS was inhibited by treatment with L-NMMA or the NO produced was scavenged by treatment with carboxy-PTIO (Fig. 2B). Treatment of cortical cultures with either L-NMMA or carboxy-PTIO during the exposure to sublethal OGD (PC) resulted in a significant decrease in the survival rate of neurons (PC NMMA + LI, PC PTIO + LI), suggesting that NO is crucially involved in the development of PC-induced ischemic tolerance in neurons. We then tried to determine whether NO was critical to the development of ischemic tolerance; that is, whether NO is produced during the period of sublethal OGD (PC) or during the period between the sublethal and lethal OGD (reperfusion period). When the production was inhibited (PC\&rep NMMA + LI) or the NO produced was scavenged (PC\&rep PTIO + LI) throughout the PC and the reperfusion period in the preconditioned cultures, a marked reduction in the survival rate of neurons was observed, 
but there was no significant difference in the survival rate of neurons as compared to the cultures with either NOS inhibition or NO scavenging only during sublethal OGD. These results suggested that NO produced during the period of sublethal OGD (PC) played a critical role in the development of neuronal torelance.

We further investigated whether the NO produced during PC is crucial to the development of PC-induced tolerance. To do this, cultures were exposed to SNAP, a donor of NO, instead of the sublethal OGD. Treatment with SNAP resulted in a significant increase in the survival rate of neurons, but that with decomposed SNAP did not (Fig. 2C).

We finally analyzed whether the NO produced during the period of lethal OGD played any role in the protection of neurons. Treatment of preconditioned cortical cultures with either L-NMMA or carboxy-PTIO during the exposure to lethal OGD resulted in an increase in the survival rate of neurons (PC + NMMA LI, PC + PTIO LI), although the increase was not significant (Fig. 3A). These results suggested that the NO produced during lethal OGD is toxic to neurons, although the NO produced during PC was crucial to the PC-induced neuronal tolerance. There is a possibility that the PC-induced increase in NOS expression was responsible for the deteriorative effect of NO on neurons during lethal OGD. Thus, 
immunohistochemical analysis was performed on the cortical cultures $24 \mathrm{hr}$ after the end of the sublethal OGD (PC) to clarify whether the expression patterns of NOS isoforms were altered by the PC insult (Fig. 3B). No detectable change in the expression patterns was observed, suggesting that NO produced by nNOS expressed in neurons was probably toxic to the neurons themselves. In fact, treatment of the non-preconditioned (control) cultures with either L-NMMA or carboxy-PTIO during the $2 \mathrm{hr}$ period of lethal OGD also increased the survival rate of neurons, although the increase was not significant (Fig. 3C). 


\section{Discussion}

The present study has demonstrated that NO produced during sublethal OGD (PC) was

crucial in triggering the signal transduction pathway responsible for the development of the PC-induced neuronal tolerance (Fig. 2). NO was probably produced by the activation of nNOS expressed in neurons. However, both the inhibition of NOS and the scavenging of NO during lethal OGD tended to increase the survival rate of neurons (Fig. 3), suggesting that NO produced during ischemia is fundamentally toxic to neurons.

A previous study (14) has revealed that the induction of neuronal ischemic tolerance is dependent on new protein synthesis, and the development of tolerance is blocked by cycloheximide. The authors postulated that the NO/p21 Ras/Raf/Erk pathway is critically involved in the PC-induced ischemic tolerance of neurons. However, it is still unclear which Erk-activated transcription factor(s) or which protein(s) regulates the neuronal tolerance (1). We have recently found that the PC-induced immediate enhancement in the phosphorylation of CREB in the penumbra region during subsequent lethal ischemia is crucially involved in the protective effects in the rat model of cerebral infarction (5). The activation of Erk has been known to stimulate nuclear transcription factors such as CREB (15). These results may 
support our previous finding that the enhanced phosphorylation of CREB is involved in the PC-induced ischemic tolerance of neurons. In the cortical cultures used here, sublethal OGD (PC) produced increased tolerance to lethal OGD (Fig. 1). However, the protective effect was transient; that is, the effects reached near maximum $24 \mathrm{hr}$ after the end of the PC insult, and decreased thereafter (Fig. 1F). Since the PC-induced protective effects were short-lived in our cultures, the possibility exists the signaling pathway triggered by NO was protein synthesis-independent, and was probably mediated by post-translational protein modification. The mechanisms of the NO-induced ischemic tolerance of neurons are now being investigated.

In the present study, both the inhibition of NOS and scavenging of NO during lethal OGD resulted in an increase in the survival rate of neurons cultured with (Fig. 3A) or without prior sublethal OGD (PC) (Fig. 3C), suggesting that the NO produced during ischemia is fundamentally toxic to neurons. The expression patterns of NOS isoforms remained unchanged by the sublethal PC insult (Fig. 3B); in particular, no marked induction of iNOS was clearly observed in neurons or astrocytes. Thus, NO produced by nNOS expressed in neurons seemed critical to the development of ischemic tolerance as well as to the neurotoxicity. The role that NO plays in the mechanisms of ischemic brain injury has long been a source of much 
debate (16). NO is known to be detrimental or beneficial to the injured brain; that is, NO functions like a double-edged sword against neurons (16). Inhibiting neuronal NOS either pharmacologically or genetically renders cultured neurons resistant to NMDA-induced death $(17,18)$, and also reduces infarct volume in rodent models of transient focal ischemia (19). However, recent studies have revealed that NO inhibits the activation of caspase-3, an enzyme crucial for apoptosis, via S-nitrosylation of the active-site cysteine of the enzyme $(20,21)$, and prevents neurons from undergoing apoptosis (22). The exact mechanism behind the PC-induced neuronal tolerance is currently unknown, but the ischemia-produced NO, fundamentally toxic to neurons, might trigger self-defense signaling in neurons against subsequent lethal ischemia.

In neuronal cells, $\mathrm{NO}$ is synthesized by neuronal $\mathrm{Ca}^{2+} / \mathrm{calmodulin}^{2}$ dependent nNOS, and is released in response to the activation of N-methyl-D-aspartate (NMDA)-type glutamate receptors $(23)$. Previous studies $(3,4)$ have found that the activation of NMDA receptors is essentiall to the PC-induced ischemic tolerance of neurons. All these findings have suggested that a sublethal ischemic insult (PC) activates neuronal NMDA receptors, and the resultant $\mathrm{Ca}^{2+}$ influx then activates nNOS. The ischemia-induced marked increase in the concentration of 
extracellular glutamate seems necessary for the excessive activation of NMDA receptors. The mechanisms responsible for this increase in glutamate are now being investigated in cortical cultures. 


\section{Acknowledgements}

The authors wish to thank Mr. Hideomi Sato of the Research Institute for Electronic Science, Hokkaido University, for help in establishing cortical cultures. 


\section{References}

1. Nandagopal, K., Dawson, T.M., and Dawson, V.L. 2002. Critical role for nitric oxide signaling in cardiac and neuronal ischemic preconditioning and tolerance. J. Pharmacol. Exp. Ther. 297:474-478.

2. Kitagawa, K., Matsumoto, M., Tagaya, M., Hara, R., Ueda, H., Niinobe, M., Handa, N., Fukunaga, R., Kimura, K., Mikoshiba, K., and Kamada, T. 1990. "Ischemic tolerance" phenomenon found in the brain. Brain Res. 528:21-24.

3. Kato, H., Liu, Y., Araki, T., and Kogure, K. 1992. MK-801, but anisomycin, inhibits the induction of tolerance to ischemia in the gerbile hippocampus. Neurosci. Lett. 139:118-121.

4. Grabb, M.C. and Choi, DW. 1999. Ischemic tolerance in murine cortical cell culture: critical role for NMDA receptors. J. Neurosci. 19:1657-1662.

5. Nakajima, T., Iwabuchi, S., Miyazaki, H., Okuma, M., Inanami, O., Kuwabara, M., Nomura, 
Y., and Kawahara, K. 2002. Relationship between the activation of CREB and ischemic tolerance in the penumbra region of rat cerebral cortex. Neurosci. Lett. 331: 13-16.

6. Ciani, E., Guidi, S., Bartesaghi, R., and Contestabile, A. 2002. Nitric oxide regulates cGMP-dependent cAMP-responsive element binding protein phosphorylation and Bcl-2 expression in cerebellar neurons: implication for a survival role of nitric oxide. J. Neurochem. 82:1282-1289.

7. Nathan, C. 1992. Nitric oxide as a secretory product of mammalian cells. FASEB J. 6:3501-3604.

8. Kawahara, K., Saitoh, M., Nakajima, T., Tanaka, M., Sato, H., Tojima, T., and Ito, E. 2002. Increased resistance to nitric oxide cytotoxicity associated with differentiation of neuroblastoma-Glioma hybrid (NG108-15) cells. Free Radical Res. 36:545-554.

9. Iwabuchi, S., Kawahara, K., Makisaka, K., and Sato, H. 2002. Photolytic flash-induced 
intercellular calcium waves using caged calcium ionophore in cultured astrocytes from newborn rats. Exp. Brain. Res. 146:103-116.

10. Kawahara, K. and Iwabuchi, S. 2003. Possible involvement of nitric oxide in the modulation of photolytic flash-induced intercellular calcium waves in cultured astrocytes. Neurosci. Res. Commun. 32:189-196.

11. Tun, H.Y., Gonzalez-Zulueta, M., Dawson, V.L., and Dawson, T.M. 1998. Nitric oxide mediates N-methyl-D-aspartate receptor-induced activation of p21ras. Proc. Natl. Acad. Sci. USA 95:5773-5778.

12. Kawahara, K., Hosoya, R., Sato, H., Tanaka, M., Nakajima, T., and Iwabuchi, S. 2002. Selective blockade of astrocytic glutamate transporter GLT-1 with dihydrokainate prevents neuronal death during ouabain treatment of astrocyte/neuron co-cultures. GLIA 40: 337-349.

13. Kawahara, K., Abe, R., Yamauchi, Y., and Kohashi, M. 2002. Fluctuations of contraction 
rhythm during simulated ischemia/reperfusion in cultured cardiac myocytes from neonatal rats.

Biol. Rhythm Res. 33: 339-350.

14. Gonzalez-Zulueta, M., Feldman, A.B., Klesse, L.J., Kalb, R.G., Dillman, J.F., Parada, L.F.,

Dawson, T.M., and Dawson, V.L. 2000. Requirement for nitric oxide activation of p21(ras)/extracellular regulated kinase in neuronal ischemic preconditioning. Proc. Natl. Acad.

Sci. USA 97:436-441.

15. Imprey, S., Obrietan, K., and Storm, D.R. 1999. Making new connections: role of ERK/MAP kinase signaling in neuronal plasticity. Neuron 23:11-14.

16. Iadecola, C. 1997. Bright and dark sides of nitric oxide in ischemic brain injury. Trends Neurosci. 20:132-139.

17. Dawson, V.L., Dawson, T.M., London, E.D., Bredt, D.S., and Snyder, S.H. 1991. Nitric oxide mediates glutamate neurotoxicity in primary cortical cultures. Proc. Natl. Acad. Sci. USA 
88:6368-6371.

18. Lee, J-M., Grabb, M.C., Zipfel, G.J., and Choi, D.W. 2000. Brain tissue responses to ischemia. J. Clin. Invest. 106:723-731.

19. Samdani, A.F., Dawson, T.M., and Dawson, V.L. 1997. Nitric oxide synthase in models of focal ischemia. Stroke 28:1283-1288.

20. Kim, Y-M., Kim, T-H., Seol, D-W., Tanalian, R.V., and Billiar, T.R. 1998. Nitric oxide suppression of apoptosis occurs in association with an inhibition of Bcl-2 cleavage and cytochrome C release. J. Biol. Chem. 273:31437-31441.

21. Mannick, J.B., Hausladen, A., Liu, L., Hess, D.T., Zeng, M., Miano, Q.X., Kane, L.S., Gow, A.J., and Stamler, J.S. 2000. Fas-induced caspase denitrosylation. Science 284:651-654.

22. Jensen, M.S., Nyborg, N.C.B., and Thomsen, E.S. 2000. Various nitric oxide donors protect 
chick embryonic neurons from cyanide-induced apoptosis. Toxicol. Sci. 58:127-134.

23. Garthwaite, J. 1991. Glutamate, nitric oxide and cell-cell signaling in the nervous system.

Trends Neurosci. 14:60-67. 


\section{Figure legends}

Fig. 1

Preconditioning (PC)-induced ischemic tolerance of neurons. Immunostaining of cultures with an anti-MAP2 antibody shows the existence of MAP2-positive neurons (A).

Photomicrographs B, C, and D show the control culture (B), and cultures exposed to oxygen-glucose deprivation (OGD) for $1 \mathrm{hr}(\mathrm{C})$, and $2 \mathrm{hr}(\mathrm{D})$. Photomicrographs C1 and D1 show the cultures before exposure to OGD, whereas C2 and D2 illustrate their state 24 hours after the exposure for 1 and $2 \mathrm{hr}$, respectively. Cell nuclei were stained with bisbenzimide (Hoechst 33342) and propidium iodide (PI) (B3, C3, and D3). Red nuclei in D3 indicate dead PI-positive neurons. Exposure to OGD for 2 hr produced significant death of cultured neurons, but exposure for1 hr did not (E). Figure $\mathrm{F}$ shows the effective time interval between the sublethal $1 \mathrm{hr}$ OGD (PC) and the lethal 2hr OGD for the development of PC-induced neuronal ischemic tolerance. The scale bar indicates $200 \mu \mathrm{m}$. Data are expressed as the mean+SD $(\mathrm{n}>4) . \quad * \mathrm{p}<0.05$. Abbreviations: rep, reperfusion (time interval between PC and LI); LI, lethal ischemia (2 hr OGD). 
Fig. 2

Nitric oxide (NO) is crucially involved in the development of preconditioning (PC)-induced neuronal ischemic tolerance. Immunocytochemical analysis using anti-nNOS (A1), anti-iNOS (A2), and anti-eNOS (A3) antibodies indicates that there were nNOS-positive neurons, but not iNOS- or eNOS-positive cells in the cortical cultures. PC-induced neuronal tolerance was significantly attenuated by treatment with either $1 \mathrm{mM}$ L-NMMA, an NOS inhibitor, or $10 \mu \mathrm{M}$ carboxy-PTIO, an NO scavenger, during sublethal OGD (PC) (B). Exposure of cultures to $100 \mu \mathrm{M}$ SNAP, a donor of NO, produced neuronal tolerance to subsequent lethal OGD, but decomposed SNAP, pre-incubated for $24 \mathrm{hr}$ to exhaust all the NO, did not (C). SNAP was dissolved in DMSO, and the final concentration of DMSO (1\%) was added to the sham control. The scale bar indicates $200 \mu \mathrm{m}$. Data are expressed as the mean+SD (n>4). $\quad * \mathrm{p}<0.05$ compared to sham. $\quad+\mathrm{p}<0.05$ compared to PC (B) or SNAP (C). Abbreviations: NMMA, L-NMMA; PTIO, carboxy-PTIO; decSNAP, decomposed SNAP. Other abbreviations are the same as those in Fig. 1. See text for details. 
Fig. 3

Nitric oxide produced during ischemia is fundamentally toxic to neurons. Treatment of preconditioned cultures with either $1 \mathrm{mM}$ L-NMMA or $100 \mu \mathrm{M}$ carboxy-PTIO during lethal OGD resulted in an increase in the survival rate of neurons (A). Immunocytochemical analysis using anti-nNOS (B1), anti-iNOS (B2), and anti-eNOS (B3) antibodies performed $24 \mathrm{hr}$ after the end of the sublethal OGD (PC) indicates that the expression patterns of NOS isoforms were essentially the same as those in the cultures before the PC insult (Fig. 2A1-A3). Treatment of non-preconditioned cortical cultures with either L-NMMA or carboxy-PTIO during lethal OGD also resulted in an increase in the survival rate of neurons, although the increase was not significant (C). The scale bar indicates $200 \mu \mathrm{m}$. Data are expressed as the mean+SD (n>4). ${ }^{*} \mathrm{p}<0.05$ compared to sham. Abbreviations are the same as those in Fig. 1 and Fig. 2. 
Abbreviations:

carboxy-PTIO,

2-(4-Carboxyphenyl)-4,4,5,5-tetramethylimidazoline-1-oxyl3-oxide; CM, conditioned medium;

CREB, cAMP-responsive element binding protein; decSNAP, decomposed

S-nitroso-N-acetyl-DL-penicillamine; eNOS, endothelial nitric oxide synthase; Erk, extracellular-signal-regulated kinase; iNOS, inducible nitric oxide synthase; L-NMMA, $\mathrm{N}^{\mathrm{G}}$-monomethyl-L-arginine; NMDA, N-methyl-D-aspartate; NO, nitric oxide; NOS, nitric oxide synthase; nNOS, neuronal nitric oxide synthase; MAP-2, microtubule-associated protein 2; OGD, oxygen and glucose deprivation; PC, preconditioning; PI, propidium iodide; SNAP, S-nitroso-N-acetyl-DL-penicillamine. 

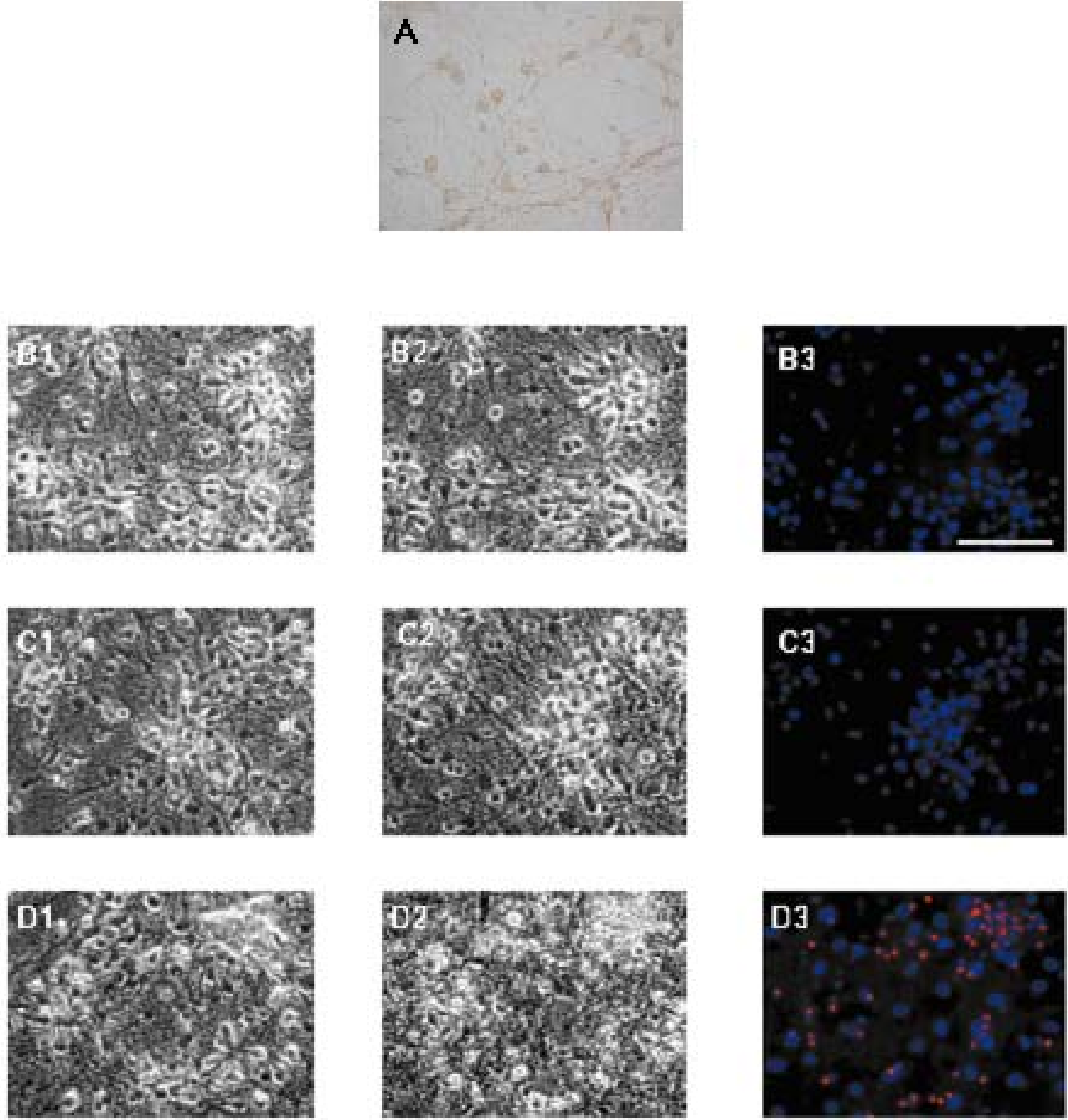

E

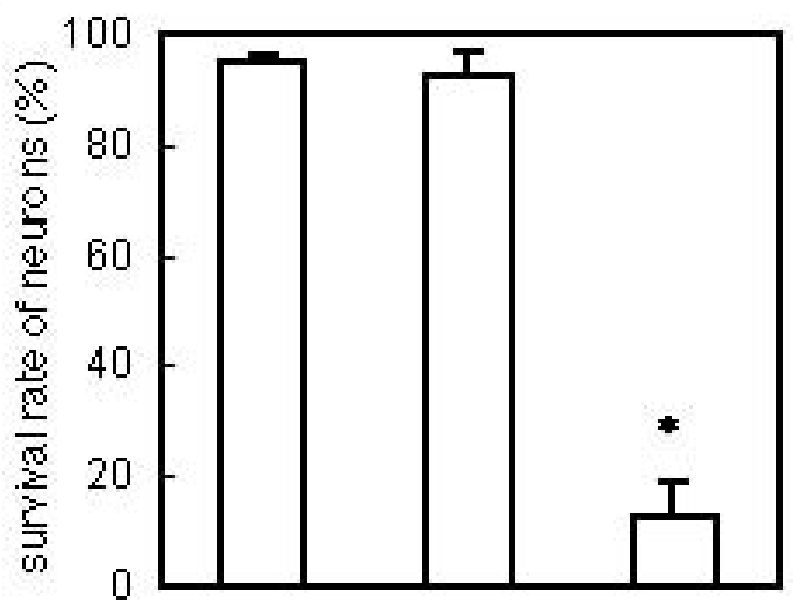

control 1hOGD 2hOGD

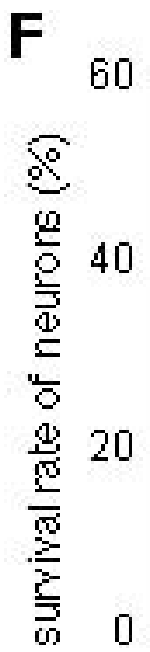

sham $\mathrm{PC} \quad \mathrm{PC} \quad \mathrm{PC}$

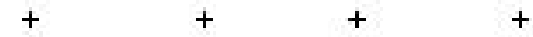

24 h rep. 6 h rep. 24 h rep. 48 h rep.

$\begin{array}{cccc}+ & + & + & + \\ \mathrm{LI} & \mathrm{LI} & \mathrm{LI} & \mathrm{LI}\end{array}$


B

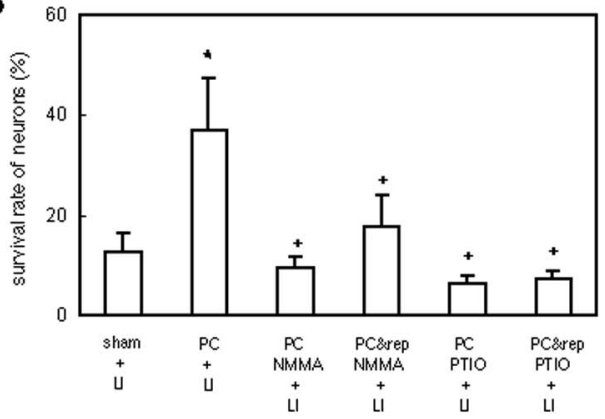

C

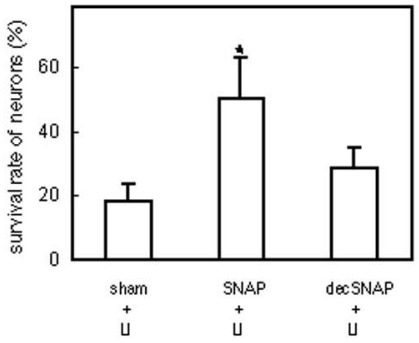



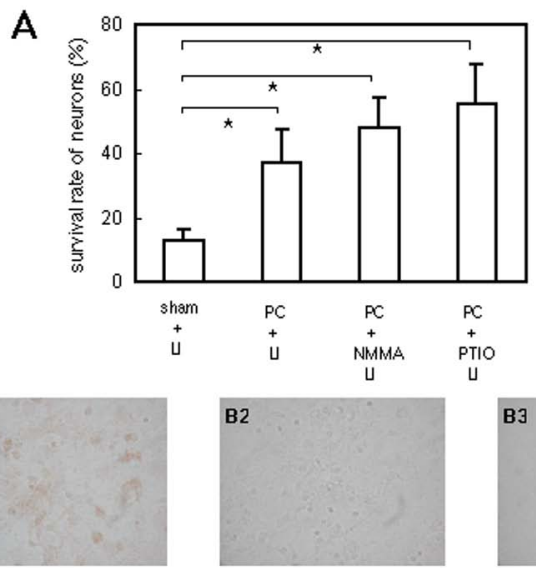

B3

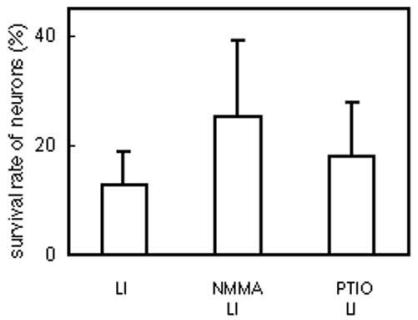

